CLAWAR 2019: 22nd International Conference on

Climbing and Walking Robots and the Support

Technologies for Mobile Machines,

Kuala Lumpur, Malaysia, 26-28 August 2019.

https://doi.org/10.13180/clawar.2019.26-28.08.12

\title{
DYNAMIC LOCOMOTION OF QUADRUPED WITH LATERALLY PARALLEL LEAF SPRING SPINE
}

\author{
HARUKI CHO, SATOSHI NISHIKAWA, RYUMA NIIYAMA, and YASUO KUNIYOSHI \\ Intelligent Systems and Informatics Laboratory, Mechano-Informatics Department of Graduate School of \\ Information Science and Technology, The University of Tokyo, Eng. Bldg.2, 7-3-1 \\ Hongo, Bunkyo-ku, Tokyo, 113-8656, Japan \\ E-mail: cho@isi.imi.i.u-tokyo.ac.jp,nisikawa@isi.imi.i.u-tokyo.ac.jp,niiyama@isi.imi.i.u-tokyo.ac.jp, \\ kuniyosh@isi.imi.i.u-tokyo.ac.jp
}

\begin{abstract}
We focus on use of the movement of the spine in animal locomotion and propose a spinal mechanism to realize dynamic spine-source locomotion. The proposed mechanism is composed of two sets of a leaf spring with a dedicated motor used to bend the spring, with these sets being placed laterally in parallel. This mechanism can realize deformation of the spine including bending and twisting and can release large amounts of elastic energy instantaneously. We equipped a quadruped robot with the proposed spinal mechanism and examined how motion changes with the motion of the spinal mechanism and legs. The robot realized rapid running up to $1.0667 \mathrm{~m} / \mathrm{s}$. The difference of the actuation between the right side and left side of the spine derived a variety of rapid and spine-source locomotion modes that changed the running direction. In addition, the timing of the simple motion of the legs further expanded the diversity of the spine-source locomotion.
\end{abstract}

\section{Introduction}

Dynamic locomotion in real environments is important subject in the research and development of quadruped robots. Drawing inspiration from the motion of animals is one of the most effective ways to realize this type of locomotion.

The dynamic locomotion of animals not only requires considerable use of the legs but also requires a great deal of movement of the body. During galloping, bending and stretching, the spine increases the stride and a sheet of tendon in the body plays a role like that of a spring that temporarily stores and releases energy. ${ }^{1}$ In the jumping of cats, the back extensor muscles of the cat make significant contributions to the overall energy production. ${ }^{2}$ In addition, the flexibility of their backs and active modulation of the bending levels of their backs allow cats to improve their energy absorption efficiency. ${ }^{3}$

However, despite the fact that animals use the movement of the body, mainstream research and development of quadruped robots in recent years has largely treated the spine as being rigid. ${ }^{4}$ In contrast to this mainstream approach, several quadruped robots with flexible spines have been researched. The MIT Cheetah ${ }^{5}$ and the Sugoi-Neco ${ }^{6}$ quadruped robots are equipped with spinal mechanisms that are mechanically linked with their legs. The Bobcat-Robot ${ }^{7}$ and the Lynx-Robot ${ }^{8}$ have dedicated actuators for their spinal mechanisms and drive them with their legs. The Cheetah-cub-S improves its steering performance by bending a spine made from a leaf spring in the yaw direction. ${ }^{9}$ Most of these quadruped robots with flexible spines treat the movement of the spine as a means to support the locomotion produced by the movement of their legs.

However, there are also quadruped robots in which the main resource for locomotion is the movement of the spinal mechanism. Kitty ${ }^{10}$ is a quadruped robot based on the theory of the spinal engine ${ }^{11}$ that runs using only the movement of a spinal mechanism that is actuated using RC motors. Laika ${ }^{12}$ is a quadruped robot that has a spinal mechanism based on tensegrity and has its legs integrated with its body. The robot locomotion provided by the movement of the spine is expected to be a useful means to realize the dynamic locomotion 


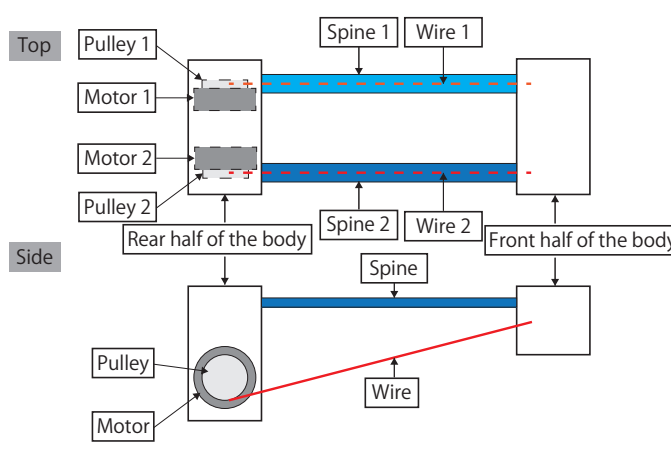

Fig. 1: Overview of spinal mechanism

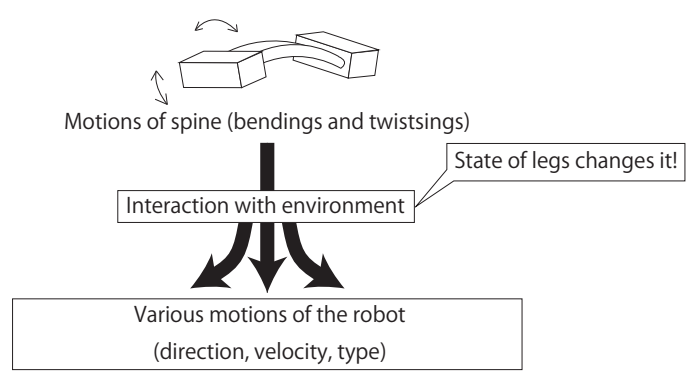

Fig. 2: Concept of the robot

of animals that make great use of the movement of their spines. We define this type of locomotion as "spine-source locomotion".

To realize the various modes of spine-source locomotion, it is necessary to move the spine in multiple directions. Therefore, the spinal mechanism requires a large number of degrees of freedom. Use of a continuum structure can enable a large number of degrees of freedom to be more simply than when using hinge joints. Continuum approaches are often focused on and used in the development of spinal mechanisms for quadruped robots that use their spines as the support for their legs. Zhang et al. showed that a spinal mechanism made from a continuum improved the performance of their quadruped robot, ${ }^{13}$ and the MIT Cheetah ${ }^{5}$ uses urethane rubber in its spinal mechanism, while the Cheetah-cub- $S^{9}$ uses a leaf spring. Kitty $^{10}$ and Laika ${ }^{12}$ both also use continua such as silicon rubber and tensegrity structures in their spinal mechanisms. However, these continua are too soft to realize the dynamic locomotion of animals in these robots.

In addition, it is important to focus on the role of the legs in spine-source locomotion to realize the dynamic locomotion modes of animals. The Stumpy Robot is a legged robot that realizes the locomotion in multiple directions using only the movement of its T-shaped upper body, which is similar to the upper body of a human, ${ }^{14,15}$ and the friction between the floor and its feet affect the stability and variety of the locomotion modes of this robot. ${ }^{16}$ It is assumed that changes in the legs play an ancillary role in changing the characteristics of spine-source locomotion even in the case of quadruped robots.

In this paper, we focus on spine-source locomotion and the changes caused by the legs to realize animal-like locomotion modes in the real world. We constructed a spinal mechanism using leaf springs for dynamic spine-source locomotion and equipped a quadruped robot with this mechanism. Then, we evaluated the variety of the spine-source running behavior of the quadruped robot and observed the changes in the running behavior caused by the movement of the simple legs when coupled with the movement of the spinal mechanism.

\section{Proposed spinal mechanism}

First, we developed the spinal mechanism to realize dynamic spine-source locomotion.

To realize various spine-source locomotion, the spinal mechanism needs deformation of spine in multiple directions. In addition, dynamic locomotion needs large acceleration. Therefore, our spinal mechanism requires,

- to be continuous

- to release large quantities of energy instantaneously

- to be lightweight.

The spinal mechanism is actuated in a dedicated manner because it needs to move actively to 


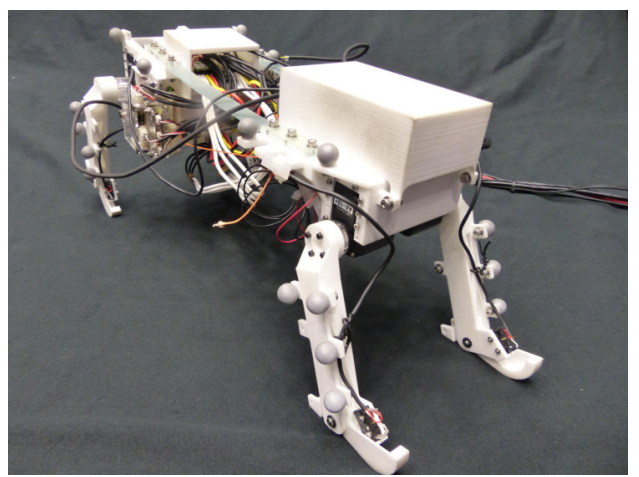

Table 1: Specification of the robot

\begin{tabular}{|c|c|}
\hline Length [m] & 0.34 \\
\hline Length of the spine [m] & 0.15 \\
\hline Width [m] & 0.2 \\
\hline Height [m] & 0.21 \\
\hline Total weight [kg] & 1.6 \\
\hline Weight of the front half of the body [kg] & 0.5 \\
\hline Weight of the rear half of the body [kg] & 1.0 \\
\hline
\end{tabular}

Fig. 3: Overview of QuaDRoPECS

enable spine-source locomotion. In addition, we use a plastic-elastic continuum in the spinal mechanism to fulfill all requirements. The plastic-elastic continuum can store and release large quantities of elastic energy. In addition, it can serve not only as an energy source but also as a structural material, thus helping the spinal mechanism to be lightweight.

When using elasticity with an actuator, the arrangement of the elasticity and the actuator is the main problem to be addressed. Parallel elasticity has a greater ability than series elasticity to store large amounts of elastic energy without an external force. However, parallel elasticity with a high-reduction-ratio actuator cannot release these large quantities of energy instantaneously because of friction losses and the counter electromotive force. Therefore, use of parallel elasticity with a high-reduction-ratio actuator requires an additional mechanism to decouple the actuator, which makes the spinal mechanism heavier and more complex. To avoid this problem, we use outer rotor brushless motors without gears in the spinal mechanism.

Figure 1 shows an overview of the spinal mechanism. This mechanism connects the front half of the body of the robot to the rear half. There are two modules arranged laterally and in parallel in the mechanism. Each module is composed of an elastic continuum and a dedicated actuator. When one actuator is driven and the other is not, a difference between the forces on the left and right generates a twist motion. However, when both actuators are driven, the mechanism can store larger quantities of energy more instantaneously. We can thus realize the variety of rapid deformations of the spinal mechanism required for spine-source locomotion.

\section{Quadruped robot design}

\subsection{Concept of the Robot}

Next, we provide a detailed overview of QuaDRoPECS (Quadrupedal Dynamic Robot with Parallel Elastic Continua Spine), a quadruped robot equipped with the proposed spinal mechanism. Figure 2 illustrates the concept of the robot. The robot treats the rapid and large-scale deformation of the spine as the main resource for locomotion and changes its locomotion with the movement of the legs. To realize this concept, the robot needs

- a spinal mechanism that is capable of rapid and large-scale deformation

- legs that support the spine-source locomotion.

The legs are simple enough to support spine-source locomotion because the main resource for the locomotion is the movement of the spine. 
Table 2: Zero positions of the angles for each leg. Forward swing is in a positive direction.

\begin{tabular}{c|cccc} 
& Right foreleg & Left foreleg & Right hind leg & Left hind leg \\
\hline Angle from body $[\mathrm{deg}]$ & 107 & 108 & 72 & 74
\end{tabular}

\subsection{Overview of the Robot}

An overview of the robot is shown in Fig. 3 and the robot's specification is given in Table 1 . The elastic continuum of the spinal mechanism is composed of a fiber-reinforced plastic (FRP) board (thickness:1.5 [mm], width:15 [mm]). The motor used for the spinal mechanism is an outer rotor brushless DC motor (Multistar Elite 3508-KV268, Turnigy) and the angle of the motor is measured using a magnetic encoder (AS5047P, ams). The motor driver (Gold Solo Twitter R50/100, Elmo) controls the angle of the motor using the signals from the encoder. The input voltage is $13.75 \mathrm{~V}$ and current limit is $10 \mathrm{~A}$. Each leg has only one degree of freedom at the shoulder and hip joint. This joint is driven using a servo motor (AX-12+, Dynamixel).

Most of the robot's structural parts are made from plastic because it is desirable that the robot is lightweight to aid in dynamic locomotion. The control board is a board computer (Raspberry Pi 3 Model B) located on the robot. Other circuit boards are also located on the robot. The power for the motors and circuits is supplied from the outside. The cables from the robots are used for power supply, operation of the control board, and sending capture start signals to the motion capture system. The motion capture system (OptiTrack, Natural Point) is used to record the robot's trajectory during locomotion.

\section{Control of spine and legs}

State machine control is used to control the robot. Each state defines the final target angle and the target angular speed for each motor in the spinal mechanism, the target angle of the servo motor for each leg, and the state transition condition. Here, the zero position for the angle of each leg is defined as Table 2 and the direction in which the leg swings forward is defined as the positive direction. With regard to the motor of the spinal mechanism, the direction in which the wire is wound is defined as positive and the zero is defined as follows.

(1) Before starting locomotion, apply 0.3 A to the motor in the direction to wind the wire

(2) Measure the angle of the motor when the wire becomes tight and define this as the zero

The control signals to the motor drivers in the spinal mechanism and to the servo motors in the legs are based on these definitions. The target angle to be sent to the motor driver in the spinal mechanism is calculated as follows.

$$
\begin{aligned}
& \theta_{\mathrm{d}}(t)=\left\{\begin{array}{lr}
\min \left(\dot{\theta_{\mathrm{d}}}\left(t-t_{\mathrm{pre}}\right)+\theta_{\mathrm{pre}}, \theta_{\mathrm{st}}\right) & \left(\dot{\theta_{\mathrm{d}}}>0\right) \\
\max \left(\dot{\theta_{\mathrm{d}}}\left(t-t_{\mathrm{pre}}\right)+\theta_{\mathrm{pre}}, \theta_{\mathrm{st}}\right) & \left(\dot{\theta_{\mathrm{d}}}<0\right) \\
\theta_{\mathrm{st}} & \left(\dot{\theta_{\mathrm{d}}}=0\right)
\end{array}\right. \\
& \dot{\theta_{\mathrm{d}}}=\operatorname{sgn}\left(\theta_{\mathrm{st}}-\theta_{\text {pre }}\right)\left|\dot{\theta_{\mathrm{d}}}\right|
\end{aligned}
$$

Here, $t$ is the time from the start of locomotion, $\theta_{\mathrm{d}}(t)$ is the target angle at $t, \dot{\theta_{\mathrm{d}}}$ is the target angular velocity, $t_{\text {pre }}$ is the time at which the previous state ends, $\theta_{\text {pre }}$ is the target angle at $t_{\text {pre }}, \theta_{\text {st }}$ is the final target angle defined in the present state, and $\left|\dot{\theta}_{\mathrm{d}}\right|$ is the target angular speed defined in the present state. The target angle that is sent to the servo motor is the same as the target angle that is defined in the state. The state transition condition is the condition where the actual angles of the motors in the spinal mechanism reach the final target angle defined in the state. 

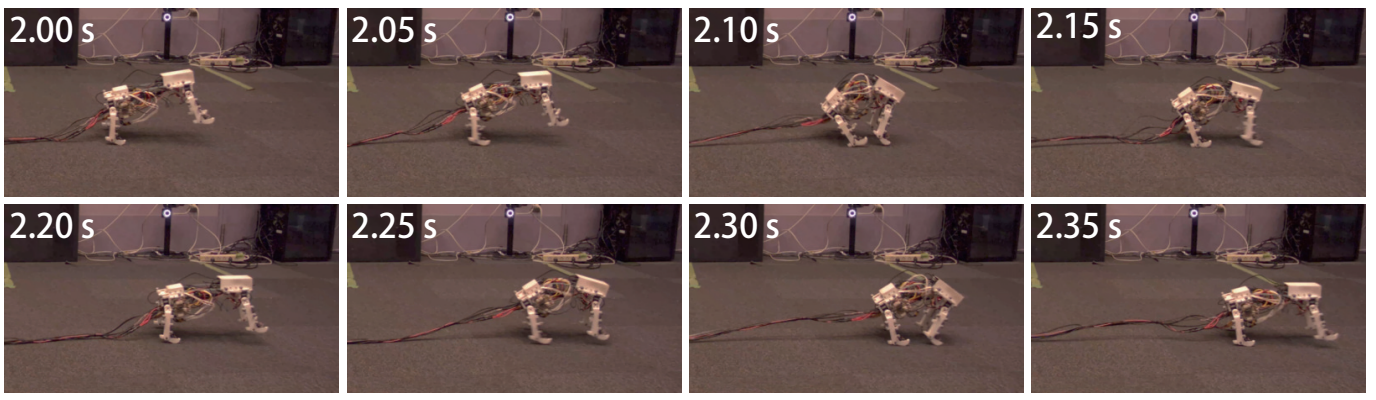

Fig. 4: Rapid spine-source running

In this paper, the controls of the motor of the spinal mechanism that are defined in each state are divided into "Wind" and "Release" controls. The motor rotates in one direction to wind the wire in Wind and rotates in the other direction in Release. The final target angles are $310 \mathrm{deg}$ in Wind and $-270 \mathrm{deg}$ in Release. The initial angles of the legs are set to zero. The commands remain at zero when the legs do not swing and the oscillation center of the command is zero when the legs swing.

\section{Experiments}

\subsection{Fast Running}

To show the physical ability of the robot, we describe the fastest running in our trials using the movement of the spinal mechanism.

The controls of both motors in the spinal mechanism were the same and repeated the Wind and Release controls. The target angular speed during Wind was $6000 \mathrm{deg} / \mathrm{s}$ and when the motor was switched from Wind to Release, the target angle was changed into the final target angle of Release in a manner similar to a step function. The commands to the legs were kept at zero.

The velocity before $2 \mathrm{~s}$ from the start of the running period varied widely, so we focused on the motion after $2 \mathrm{~s}$. The average velocity of the rear half of the body when moving in the forward direction from the first Wind after $2 \mathrm{~s}$ to the end of four periods was $1.0667 \mathrm{~m} / \mathrm{s}$. We observed the spine-source bounding like that in Fig. 4.

\subsection{Variation in the spine-source running}

We examined how the spine-source locomotion behavior changes with the changes in the control of the spine mechanism. We observed how the difference of actuation between right motor and left motor in the spine mechanism and the speed of the motion of the spine mechanism affect the locomotion.

We tried two modes of control for the spine mechanism: the right motor repeating Wind and Release while the left maintained Release ("Right"), and the left motor repeating Wind and Release while the right maintained Release ("Left"). We tried three values for the target angular speed in Wind and Release (4000, 6000, and $8000 \mathrm{deg} / \mathrm{s}$ ) in each control mode. We also tried to make rapid changes in the final target angle, like a step function, when switching control mode of spine. The commands to the legs were maintained at zero. We observed the locomotion for $3 \mathrm{~s}$ after $1 \mathrm{~s}$ had passed from the start of the locomotion period. This is because the locomotion for the first second after starting locomotion varied widely.

We defined the position of pseudo center of gravity ( $\mathrm{pCoG}$ ) as the weighted mean of pCoGs of front and rear half of the bodies. We calculated pCoG of each part as the average of four markers on it. Figure 5 shows the trajectories of pCoG on top view in Right modes 


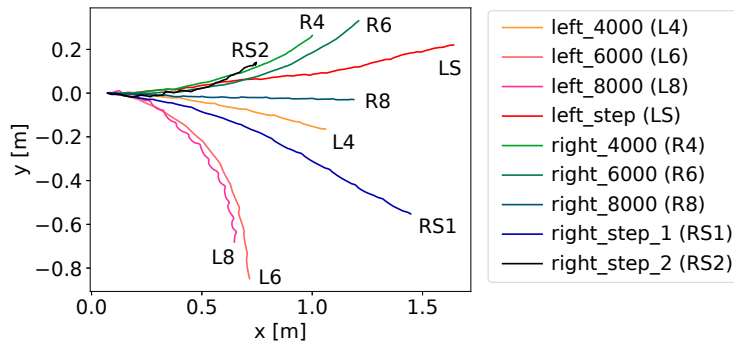

Fig. 5: Trajectories of $\mathrm{pCoG}$ on top view

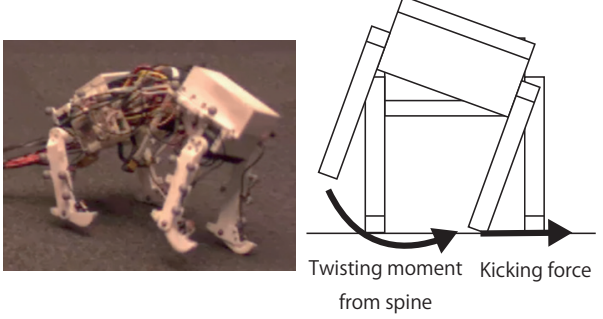

Fig. 6: Touchdown during running $\{$ Left, $8000 \mathrm{deg} / \mathrm{s}\}$

and Left modes. Here, the x-axis positive direction is regarded as the robot's front direction at $t=1 \mathrm{~s}$ in each locomotion.

Running to the left was observed in the most Right control mode and running to the right was observed in the most Left control mode. It is assumed that this occurs because a difference in the moving distance between the right and left sides of the robot was generated by the difference between the force on the right side of the spinal mechanism and that on the left side.

However, running to the right was observed in \{Right, step-change (right_step_1 in Fig. 5) and running to the left was observed in \{Left, step-change (left_step in Figure 5). These characteristics differ from the characteristics explained above. It is assumed that the rapid and asymmetric actuation of the spine made the spine twist, and this twist then generated locomotion in which the robot mainly kicked the ground with its right foreleg and left hind leg in the Right control mode but mainly kicked the ground with its left foreleg and right hind leg in the Left control mode.

In the Right control mode, the trajectory approached that of a step-change as the target angular speed increased, except for that of right_step_2. This indicates a transition from locomotion to the left due to the force difference into locomotion to the right caused by the twist. In right_step_2, the robot ran to the right like the locomotion of right_step_1 at the beginning and ran to the left after that. Therefore, it is assumed that the trajectory of right_step_2 was caused by the combination of the effect of the force difference and the effect of the twist.

In the Left control mode, the robot ran further to the right as the target angular speed increased except in the step-change case. During locomotion of $\{$ Left, $8000 \mathrm{deg} / \mathrm{s}\}$, the twist in the spinal mechanism was observed but the locomotion characteristics differed from those of the step-change case. During locomotion of $\{\mathrm{Left}, 8000 \mathrm{deg} / \mathrm{s}\}$, we observed a phenomenon where the left foreleg landed on the outside of the foot, as shown in Fig. 6, and then kicked the ground with the rebound from the twist as the front half of the body moved toward the right. It is assumed that this phenomenon made the robot run further to the right.

\subsection{Change via the motion of the legs}

We examined how the spine-source locomotion process changes with movement of the legs. We observed how the timing of the swing of the leg affect the spine-source locomotion.

The controls of both motors in the spinal mechanism were the same and repeated the Wind and Release modes. The target angular speed in the Wind and Release mode was $4000 \mathrm{deg} / \mathrm{s}$. The commands to the legs were changed in accordance with the changes in the commands to the spinal mechanism. The amplitude of the swing of the legs was $\pm 7.54 \mathrm{deg}$. We defined command of $t_{\text {diff }}=0 \mathrm{~s}$ as the command to the legs that the legs would swing inside the body when bending the spine and would swing outside the body when stretching 


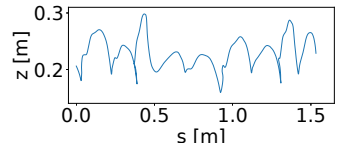

(a) $t_{\text {diff }}=0.0 \mathrm{~s}$

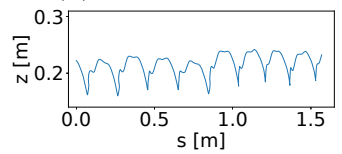

(e) $t_{\text {diff }}=0.1 \mathrm{~s}$

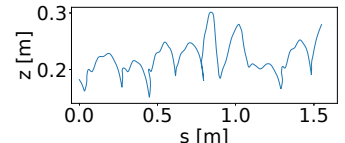

(b) $t_{\text {diff }}=0.025 \mathrm{~s}$

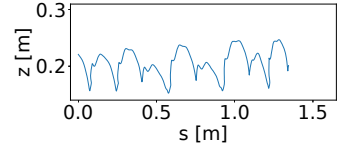

(f) $t_{\text {diff }}=0.125 \mathrm{~s}$

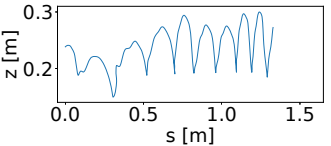

(c) $t_{\text {diff }}=0.05 \mathrm{~s}$

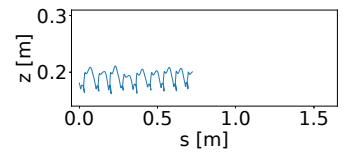

(g) Anti-phase

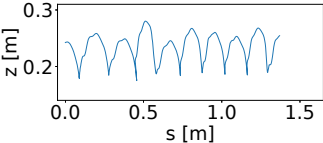

(d) $t_{\text {diff }}=0.075 \mathrm{~s}$

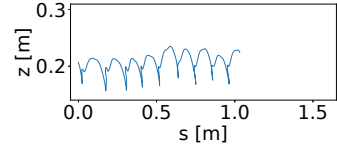

(h) Not swinging

Fig. 7: Trajectory of front half of the body ( $s$ : distance when moving forward, $z$ : height)

the spine. When the switching command for the target angle of the legs is $t$ seconds behind the change in the commands to the spinal mechanism, $t_{\text {diff }}=t \mathrm{~s}$. We tried commands of $t_{\text {diff }}=\{0,0.025,0.05,0.075,0.1,0.125\} \mathrm{s}$ and anti-phase commands that were the opposites to the commands for $t_{\text {diff }}=0 \mathrm{~s}$. We also tried the command maintaining the angles of the legs at zero. We observed the resulting locomotion for $3 \mathrm{~s}$ after $1 \mathrm{~s}$ had passed from the start of the locomotion.

Figure 7 shows the trajectory of the front half of the body. The horizontal axis shows the distance when moving forward and the vertical axis shows the height. When $t_{\mathrm{diff}}=$ $\{0,0.025,0.05\} \mathrm{s}$, the robot moved upward and there was a large difference of the shape of the trajectory among the periods. When $t_{\text {diff }}=0.075 \mathrm{~s}$, the difference of the shape of the trajectory among the periods was not large but the robot moved further upward than when the legs did not swing. When $t_{\text {diff }}=0.1 \mathrm{~s}$, the difference of the shape of the trajectory among the periods was small and the robot did not move upward much. When $t_{\text {diff }}=0.125 \mathrm{~s}$, the robot did not move upward much but two types of motion were observed alternately for each period. When the anti-phase commands were sent, small stride-type locomotion was observed. It is assumed that this occurs because the swing of the legs offset the propulsive force that was generated by the spinal mechanism.

In Fig. 7, the trajectory of one kind of motion observed when $t_{\text {diff }}=0.125 \mathrm{~s}$ was similar to the trajectory when $t_{\text {diff }}=0.1 \mathrm{~s}$ in shape and height while other was similar to that of the anti-phase in shape and height. It is assumed that the appearance of two types of the motion when $t_{\text {diff }}=0.125 \mathrm{~s}$ was a result of a transition in the characteristics of the locomotion between $t_{\text {diff }}=0.1 \mathrm{~s}$ and the anti-phase.

\section{Conclusions}

In this paper, we have proposed the spinal mechanism to realize spine-source locomotion that uses laterally parallel leaf springs and deforms the springs using motors. We then constructed a quadruped robot that was equipped with the proposed mechanism and observed this robot's while it was running. First, we confirmed that the proposed mechanism realized rapid spine-source running up to $1.0667 \mathrm{~m} / \mathrm{s}$ by rapidly repeating bending and stretching motions. Next, we examined how motion changes using the spine mechanism. We found that the traveling direction was changed by the speed of either actuation of the right or left side of the mechanism. The direction tendency was reversed at some speed even if the actuation side was the same. This result suggests that the directional change of the proposed robot could be induced by multiple principles. It indicates that the proposed spine mechanism has the potential to generate various motion patterns. Finally, we investigated the motion variation caused by combining the leg motion with spine-source running. Here, we confirmed that the degree of the phase difference between spine and leg motion affects 
locomotion patterns. These results show that the proposed spinal mechanism can realize rapid and varied locomotion and simple use of the legs can further expand the diversity of this spine-source locomotion.

In future work, an additional mechanism to bend the leaf spring upward should be added to realize more variations in the locomotion. In addition, use feedback measures such as the position, posture and floor reaction force to aid in control of the robot is also desirable.

\section{Acknowledgments}

This work was supported by JSPS KAKENHI under Grant No. JP18H05466 and JP18K18087.

\section{References}

1. R. M. Alexander, Exploring Biomechanics: Animals in Motion (Scientific American Library, New York, 1992).

2. T. H. Harty, The role of the vertebral column during jumping in quadrupedal mammals, $\mathrm{PhD}$ thesis, Oregon State University2010.

3. Z. Zhang, J. Yang and H. Yu, Journal of Bionic Engineering 11, 506(oct 2014).

4. M. Hutter, C. Gehring, D. Jud, A. Lauber, C. D. Bellicoso, V. Tsounis, J. Hwangbo, K. Bodie, P. Fankhauser, M. Bloesch et al., Anymal-a highly mobile and dynamic quadrupedal robot, in IROS, 2016.

5. S. Seok, A. Wang, M. Y. Chuah, D. Otten, J. Lang and S. Kim, Design principles for highly efficient quadrupeds and implementation on the MIT Cheetah robot, in ICRA, 2013.

6. R. Kawasaki, R. Sato, E. Kazama, A. Ming and M. Shimojo, Development of a flexible coupled spine mechanism for a small quadruped robot, in $R O B I O, 2016$.

7. M. Khoramshahi, A. Spröwitz, A. Tuleu, M. N. Ahmadabadi and A. J. Ijspeert, Benefits of an active spine supported bounding locomotion with a small compliant quadruped robot, in ICRA, 2013.

8. P. Eckert, A. Spröwitz, H. Witte and A. J. Ijspeert, Comparing the effect of different spine and leg designs for a small bounding quadruped robot, in ICRA, 2015.

9. K. Weinmeister, P. Eckert, H. Witte and A.-J. Ijspeert, Cheetah-cub-s: Steering of a quadruped robot using trunk motion, in SSRR, 2015.

10. Q. Zhao, H. Sumioka and R. Pfeifer, The effect of morphology on the spinal engine driven locomotion in a quadruped robot, in $A M A M, 2011$.

11. S. Gracovetsky, Journal of biomedical engineering 7, 205 (1985).

12. A. P. Sabelhaus, L. J. van Vuuren, A. Joshi, E. Zhu, H. J. Garnier, K. A. Sover, J. Navarro, A. K. Agogino and A. M. Agogino, CoRR abs/1804.06527 (2018).

13. X. Zhang, H. Yu, B. Liu and X. Gu, A bio-inspired quadruped robot with a global compliant spine, in $R O B I O, 2013$.

14. F. Iida, R. Dravid and C. Paul, Design and control of a pendulum driven hopping robot, in IROS 2002.

15. C. Paul, R. Dravid and F. Iida, Control of lateral bounding for a pendulum driven hopping robot, in CLAWAR, 2002.

16. F. Iida, Exploiting friction for the locomotion of a hopping robot, in $A M A M, 2003$. 